\title{
Erratum to: Abiogenic Photophosphorylation of ADP to ATP Sensitized by Flavoproteinoid Microspheres
}

\author{
Michael P. Kolesnikov ${ }^{1}$ Taisiya A. Telegina ${ }^{1}$ • \\ Tamara A. Lyudnikova ${ }^{1}$ - Mikhail S. Kritsky ${ }^{1}$
}

Published online: 28 April 2015

(C) Springer Science+Business Media Dordrecht 2015

\section{Erratum to: Orig Life Evol Biosph (2008) 38(2):243-255 \\ DOI 10.1007/s11084-008-9130-9}

Three coauthors of this previously published work Taisiya A. Telegina, Tamara A. Lyudnikova and Mikhail S. Kritsky wish to retract Figs. 1, 2, 3, 4 and Table 1 from our paper. The retraction involves the content of the paper claiming to show that illumination of the aqueous solution of ADP and $\mathrm{K}_{2} \mathrm{HPO}_{4}$ in presence of flavoproteinoid microspheres leads to formation of ATP. We have no reason to believe that the other results and interpretations presented in our paper are in error. This retraction follows a thorough investigation performed in the Bach Institute of Biochemistry into the scientific misconduct of the fourth of the original authors of this work, Dr. Michael P. Kolesnikov, who is no longer a member of the Institute staff. The investigation found that Dr. Kolesnikov was solely responsible for the falsified or fabricated data presented in the cited figures and table that resulted in the unsubstantiated conclusions presented.

The online version of the original article can be found at 10.1007/s11084-008-9130-9.

Mikhail S. Kritsky

mkritsky@inbi.ras.ru

Taisiya A. Telegina

telegina@inbi.ras.ru

Tamara A. Lyudnikova

lyudnikova@inbi.ras.ru

1 Bach Institute of Biochemistry of the Russian Academy of Sciences, 33(bldg 2) Leninsky Prospekt, Moscow 119071, Russia 